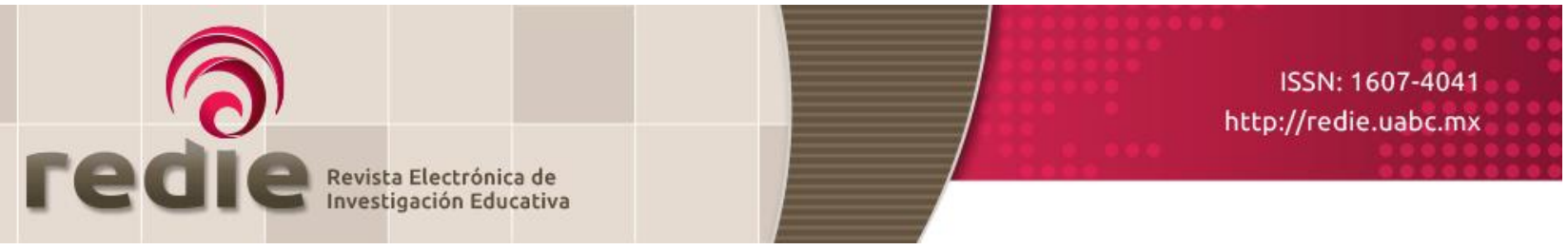

Vol. 20, Núm. 1, 2018

\title{
La formación intercultural de los futuros maestros mexicanos de secundaria
}

\section{Intercultural Training of Future Mexican Junior High School Teachers}

\author{
Andreas Pöllmann (*) andreas.poellmann@phil.tu-chemnitz.de \\ (*) Chemnitz University of Technology \\ (Recibido: 23 de junio de 2016; Aceptado para su publicación: 8 de diciembre de 2016)
}

Cómo citar: Pöllmann, A. (2018). La formación intercultural de los futuros maestros mexicanos de secundaria. Revista Electrónica de Investigación Educativa, 20(1), 83-92. https://doi.ora/10.24320/redie.2018.20.1.1461

\section{Resumen}

La provisión de oportunidades para el aprendizaje intercultural a través del contacto con "lo extranjero" es fundamental para el bienestar cultural, social y económico de los países multiculturales. El contexto mexicano es de particular interés tanto por su extraordinaria diversidad sociocultural como por sus niveles epidémicos de analfabetismo intercultural. Con base en una encuesta realizada a 189 estudiantes de la Escuela Normal Superior de México, el presente artículo explora (i) la importancia percibida del contacto con "lo extranjero" como parte de la formación magisterial, (ii) el interés existente por la participación en algún programa de intercambio internacional y (iii) el nivel de experiencias concretas con "lo extranjero". A partir de una serie de estadísticas descriptivas -que demuestran las percepciones, intereses y experiencias de los futuros maestros de secundaria- se manifiesta la importancia de un mayor compromiso institucional y gubernamental con la formación intercultural del magisterio normalista.

Palabras clave: Educación secundaria, formación intercultural, maestros, México.

\begin{abstract}
Opportunities for intercultural learning through contact with "foreignness" are fundamental for the cultural, social, and economic well-being of multicultural countries. The Mexican context is of particular interest, both due to its extraordinary socio-cultural diversity and its epidemic levels of intercultural illiteracy. Based on an survey conducted with 189 students of the Teacher Training College of Mexico City (Escuela Normal Superior de México), this study explores (i) the perceived importance of contact with "foreignness" as part of teacher training, (ii) existing interest in participating in an international exchange program, and (iii) the level of actual experiences with "foreignness". A series of descriptive statistics which show the perceptions, interests, and experiences of future junior high school teachers demonstrates the importance of greater institutional and governmental engagement toward intercultural training in teacher colleges.
\end{abstract}




\section{Introducción}

El contacto con "lo extranjero", "lo desconocido" y "el otro" son claves para una mejor comprensión del mundo globalmente interconectado en el que vivimos; son vitales para la vida tanto de individuos como de sociedades - para su prosperidad económica, social y cultural. El desarrollo socioculturalmente justo e incluyente de comunidades, regiones y naciones enteras depende de la provisión de oportunidades para el aprendizaje intercultural. En consecuencia, se están buscando maneras de construir y mantener sistemas de educación formal que reconozcan y valoren la diversidad humana (Leiva, 2006; Pérez-Silva y Luis, 2015; Pöllmann, 2009, 2014, 2016; Villalta, 2016).

Efectivamente, en muchas de las sociedades multiculturales contemporáneas, el tema de la educación intercultural ocupa un lugar preponderante en la consciencia política, pedagógica y sociocultural, y se están realizando investigaciones sobre la construcción y expansión de entornos escolares y universitarios interculturales. Los contextos nacionales de Argentina (Hecht, 2015), Australia (Santoro, 2014), Bolivia (Balda, 2015), Brasil (Paladino, 2015), Chile (Turra y Ferrada, 2016) y México (Fernández, Torres y García, 2016) se podrían mencionar como ejemplos, entre muchos otros.

En el caso de México, en el que se concentra este artículo, el Artículo 2 de la constitución política vigente reconoce explícitamente el carácter pluricultural del país (Constitución Política de los Estados Unidos Mexicanos, 2016). A pesar de la importancia fundamental de tal reconocimiento, éste no es suficiente para entender el estado de las relaciones interculturales fácticas o vividas por la población nacional, por lo que vale la pena considerar algunos de los resultados recientes de la Encuesta Nacional de Cultura, Lectura y Deporte (Pöllmann y Sánchez-Graillet, 2015) que documentan un rango de percepciones, experiencias y actitudes altamente pertinentes para los campos de la educación intercultural -entre los cuales destaca el fenómeno sociocultural epidémico del "analfabetismo intercultural".

Dicho analfabetismo intercultural se manifiesta, por un lado, en el hecho empírico de que la gran mayoría de los encuestados no tiene amigos indígenas y "de que más de 9 de cada 10 encuestados no [son] capaces de tener una conversación básica, ni de leer un texto en algún idioma indígena del país" (Pöllmann, 2015, p. 158). Por otro lado -y de particular interés en el contexto del presente artículo- llama la atención que una mayoría significativa de los encuestados nunca haya vivido en el extranjero, no tenga amigos extranjeros y no hable ni lea en algún idioma extranjero (Pöllmann y Sánchez-Graillet, 2015).

Teniendo lugar el escenario de un analfabetismo intercultural prevaleciente, no parece utópico sugerir que el futuro bienestar cultural, social y económico de los Estados Unidos Mexicanos dependerá en gran medida de inversiones sustanciales en entornos de diálogo y aprendizaje interculturales. Como lo demuestra la Encuesta Nacional de Cultura, Lectura y Deporte de manera reveladora, tales inversiones podrían contar con un considerable respaldo de la opinión pública. Por ejemplo, es notable que la mayoría de las personas encuestadas "hayan atribuido cierta o mucha importancia al planteamiento de que los maestros de primaria tengan la posibilidad de conocer culturas y gente de otros países." (Pöllmann, 2015, p. 155).

Рага сrear y cultivar entornos escolares de diálogo y aprendizaje interculturales socioculturalmente justos e incluyentes, los sistemas educativos correspondientes necesitan tomar en cuenta los puntos de vista de los (futuros) maestros y construir -a partir de sus percepciones, intereses y experiencias-oportunidades de formación intercultural inicial y continua. En este espíritu, y con base en una encuesta a estudiantes de la Escuela Normal Superior de México (ENSM) -la principal institución formadora de maestros de educación secundaria del país (Ducoing, 2007, 2013)- la presente investigación busca respuestas a las siguientes preguntas: 1) ¿Qué importancia atribuyen los futuros maestros al contacto con "lo extranjero" como parte de la formación magisterial? 2) ¿Qué interés tienen los futuros maestros en participar en algún programa de intercambio internacional?, y 3) ¿Cuáles han sido sus experiencias con "lo extranjero" en el contexto de su formación magisterial?

Antes de exponer los datos cuantificados que dan respuestas empíricamente fundamentadas a estas tres preguntas de investigación, se presentan algunas características contextuales y metodológicas de la encuesta realizada a los maestros en formación. 


\section{Método}

Conforme al reconocimiento constitucional de la pluriculturalidad de los Estados Unidos Mexicanos, el programa de la Licenciatura en Educación Secundaria, vigente en la ENSM, exige un perfil del futuro magisterio que respete apreciativamente "la diversidad regional, social, cultural y étnica del país como un componente valioso de la nacionalidad (...) que (...) estará presente en las situaciones en las que realice[n] su trabajo" (Secretaría de Educación Pública, 2000, p. 13).

Sin embargo, hasta la fecha, estos reconocimientos oficiales no han sido traducidos de manera clara y contundente en los programas de formación de maestros de secundaria. De hecho, el modelo curricular de la Licenciatura en Educación Secundaria antes mencionado no se refiere de manera explícita a oportunidades concretas de formación intercultural, no se hace referencia a los contactos con pueblos y comunidades indígenas dentro del país, ni tampoco a los intercambios educativos internacionales, los cuales ya constituyen parte integral de los programas de formación magisterial en muchos países miembros de la Organización para la Cooperación y el Desarrollo Económicos (OCDE).

Con el fin de ofrecer una mirada que vaya más allá del estudio de (la ausencia de) lineamientos formales, el presente artículo investiga la formación intercultural inicial y continua de los maestros de educación secundaria desde la perspectiva de los estudiantes de la ENSM. Se diseñó un cuestionario impreso para ser completado en un tiempo aproximado de 35 minutos, como se pudo estimar a partir de las pruebas piloto realizadas con estudiantes de la Universidad Nacional Autónoma de México con un perfil académico comparable al de los estudiantes de la Escuela Normal Superior. La versión final del cuestionario consta de 47 preguntas operacionales, algunas de las cuales contienen sub-preguntas con varios elementos a evaluar. ${ }^{1}$

El trabajo de campo se efectuó en las últimas dos semanas de septiembre y la primera semana de octubre de 2014 durante el turno matutino en las instalaciones de la ENSM. Se distribuyeron cuestionarios a estudiantes de tercero y quinto semestre ${ }^{2}$ de la Licenciatura en Educación Secundaria que cursaban las siguientes especialidades: Formación cívica y ética, Historia, Pedagogía, Español y Matemáticas. A partir de la participación de todos los estudiantes presentes en los respectivos salones de clase, se obtuvo un total de 189 cuestionarios llenos, lo que condujo a una base de datos de alta calidad. ${ }^{3}$

De los 189 participantes, 90 cursaban el tercer semestre y 99 el quinto semestre. Cerca de cuatro de cada cinco estudiantes encuestados fueron mujeres (78.3 por ciento). Todos los participantes nacieron en el mismo país (México) e indicaron no tener otra nacionalidad. 23 (12.2\%) se identificaron como indígenas y 166 (87.8\%) como no indígenas. La edad promedio de los estudiantes encuestados fue de 21.1 años. Cabe mencionar que participaron 12 estudiantes de la especialidad en Formación cívica y ética (6.3\%), 20 de Pedagogía (10.6\%), 24 de Historia (12.7\%), 65 de Matemáticas (34.4\%) y 68 de Español (36.0\%) -lo que refleja la importancia de la matrícula de las distintas áreas del conocimiento en la ENSM.

\section{Resultados}

Con base en lo expuesto, esta sección presenta los hallazgos empíricos más pertinentes, considerando: 1) la importancia percibida del contacto con "lo extranjero" como parte de la formación magisterial, 2) el interés existente por la participación en algún programa de intercambio internacional y 3) el nivel de experiencias concretas con "lo extranjero" en el contexto de la formación magisterial.

\footnotetext{
${ }^{1}$ Copias del cuestionario y de la base de datos (en formato SPSS) se pueden obtener previa solicitud escrita, debidamente firmada y dirigida al autor de este artículo.

${ }^{2}$ Dado a que sólo hay una fecha de ingreso a la ENSM, y a que la investigación tuvo lugar en los meses de septiembre-octubre, sólo se encontraban en curso los semestres nones (primero, tercero, quinto y séptimo). El primer semestre deliberadamente no fue considerado porque los alumnos de ese semestre no contaban con la experiencia necesaria para contestar muchas de las preguntas del cuestionario. Por otro lado, el séptimo semestre no se consideró debido a que los estudiantes de ese semestre se encontraban realizando prácticas en distintas escuelas, lo que no garantizaba que fueran incluidos de manera representativa en la encuesta.
}

${ }^{3}$ Cabe señalar que no hubo problemas con la comprensión de las respectivas preguntas, ni tampoco con el manejo de los distintos formatos de respuesta. 


\subsection{La importancia percibida del contacto con "lo extranjero" como parte de la formación magisterial}

Como tendencia general, destaca la alta importancia que los estudiantes normalistas dieron al contacto con "lo extranjero" como parte de la formación magisterial -tanto en la formación inicial como en la formación continua. En términos más específicos, se observan altos niveles de importancia asignados al contacto con los sistemas educativos (figura 1), los maestros (figura 2) y las culturas (figura 3) de otros países.

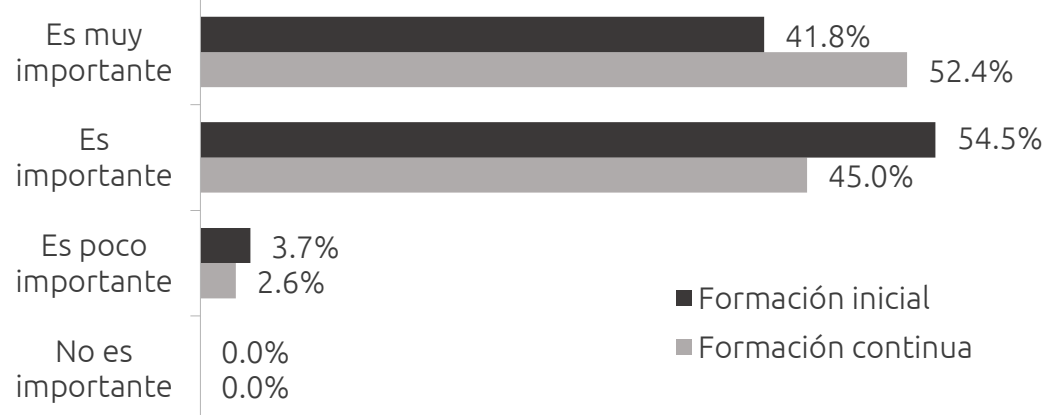

Figura 1. Importancia atribuida al contacto con sistemas educativos de otros países

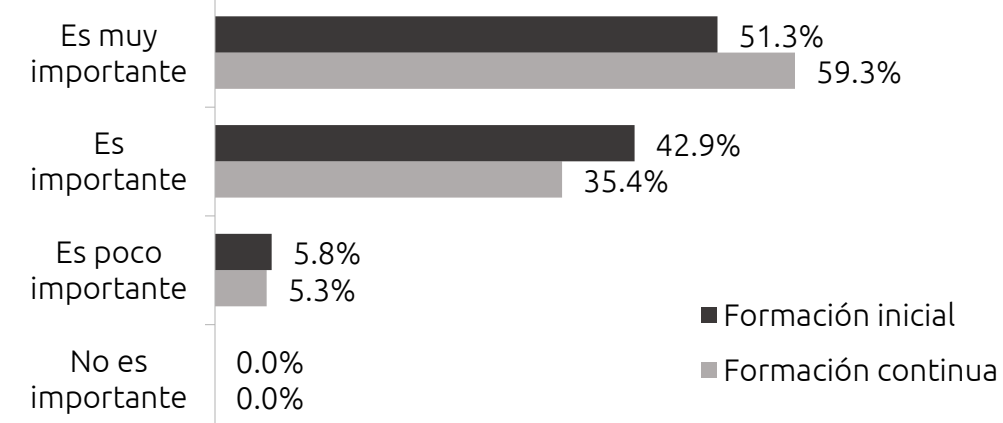

Figura 2. Importancia atribuida al contacto con maestros de otros países

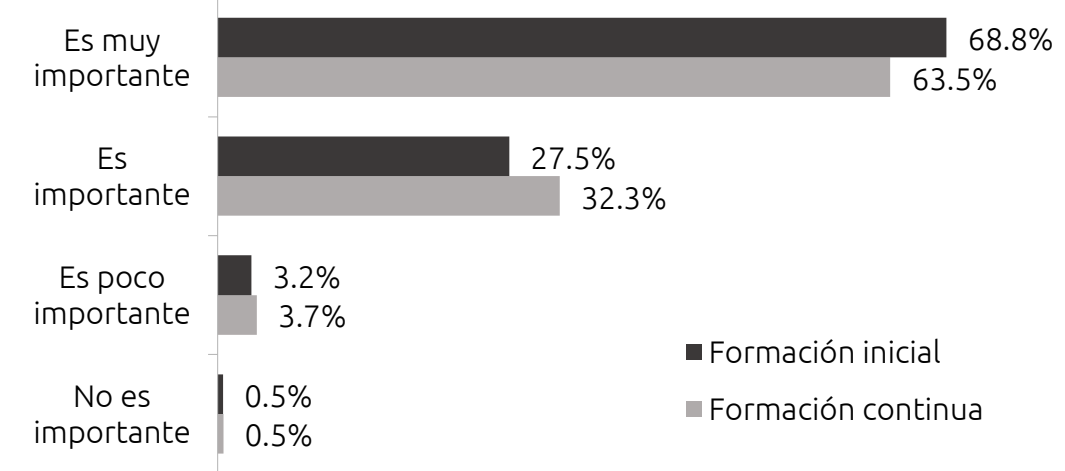

Figura 3. Importancia atribuida al contacto con culturas de otros países 
También son notables los altos niveles de importancia atribuidos a la posibilidad de poder enseñar en otros países (figura 4).

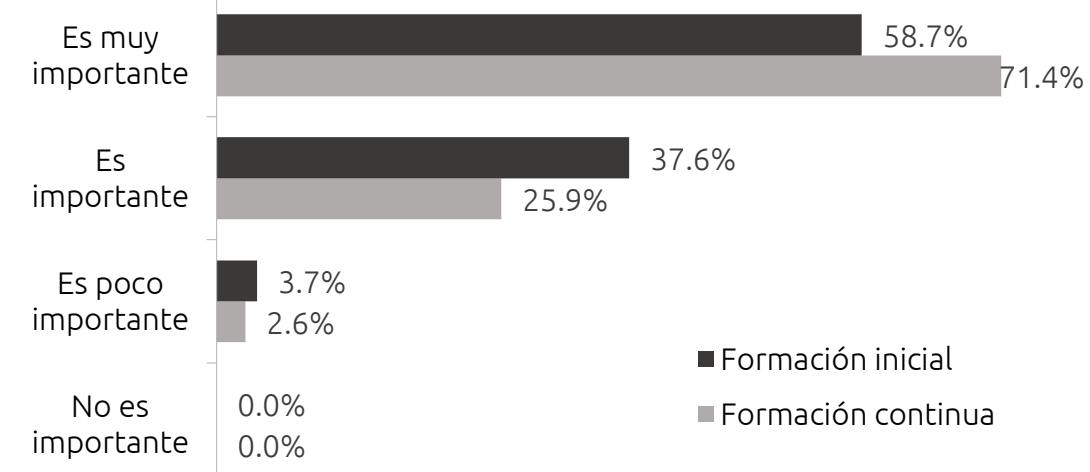

Figura 4. Importancia atribuida a la experiencia de enseñanza en otros países

Al revisar los resultados expuestos en las figuras 1 a la 4, llama la atención que las categorías de respuesta "es importante" y "es muy importante" combinadas representan, respectivamente, entre el $94.2 \%$ y el $97.4 \%$ de las respuestas de los estudiantes normalistas. Además, vale la pena señalar que, con excepción del contacto con culturas de otros países (figura 3), los distintos niveles de importancia asignados son comparativamente más elevados respecto al ámbito de la formación continua de maestros, lo que sugiere que muchos de los encuestados conciben el aprendizaje intercultural como algo procesual y duradero.

\subsection{Interés por participar en algún programa de intercambio internacional}

En cuanto al interés por participar en algún programa de intercambio internacional, se consideró en primer lugar a los países hispanohablantes. Como lo demuestra la figura 5, más de 7 de cada 10 estudiantes normalistas encuestados dijeron estar "muy interesados" en ese tipo de intercambio, mientras que ninguno eligió la categoría "nada interesado".

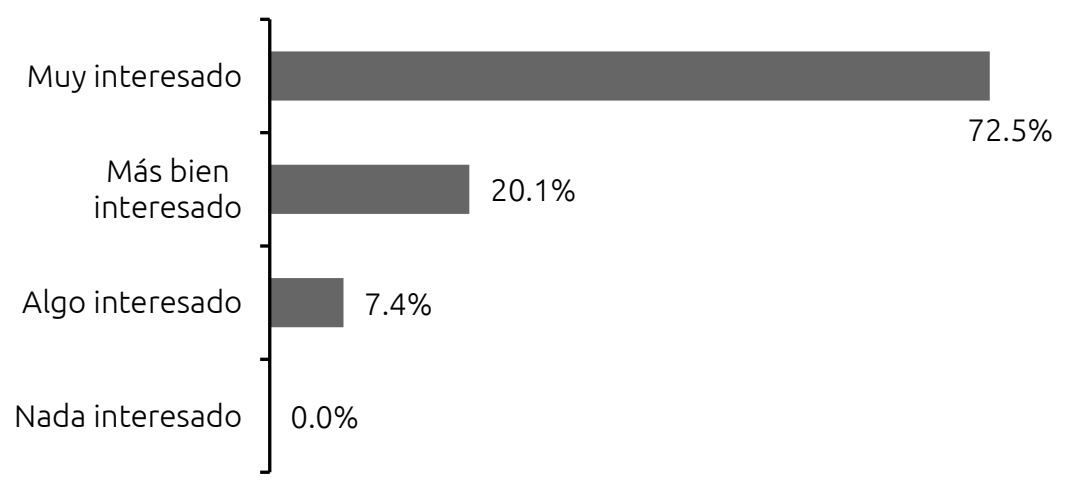

Figura 5. Interés por el intercambio con países hispanohablantes 
Entre los países hispanohablantes mencionados por los encuestados (en forma de respuesta abierta), se encuentra España en primer lugar con el $51.3 \%$ de las menciones; seguido por Argentina (15.3\%), Cuba (13.2\%) y otros países con porcentajes de respuesta menos significativos. La figura 6 muestra la representación gráfica de la distribución proporcional global.

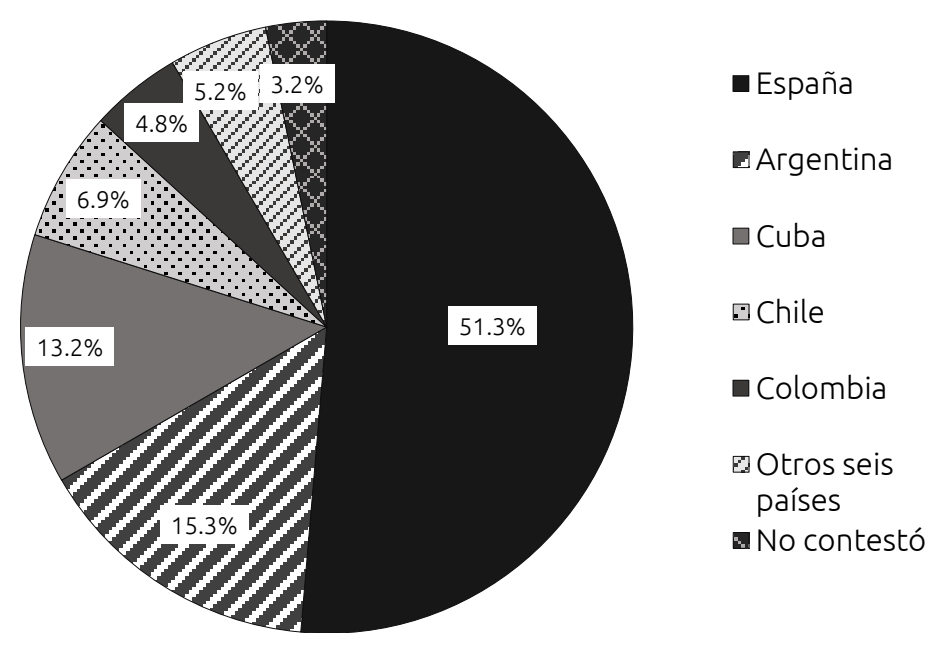

Figura 6. País hispanohablante preferido como primera opción

Como lo señala la figura 7, los niveles de interés por participar en algún programa de intercambio internacional en países no-hispanohablantes son inferiores a los observados anteriormente para los países en donde el español tiene el estatus de lengua oficial. Sin embargo, considerando el esfuerzo necesario para aprender un idioma extranjero, es notable que más que 8 de cada 10 estudiantes indicaran estar "muy interesados" (55\%) o "más bien interesados" (27.5\%).

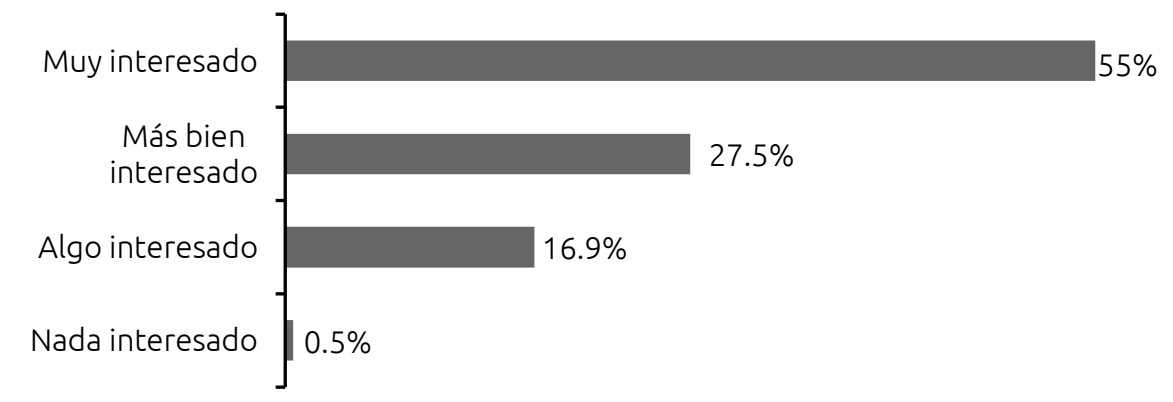

Figura 7. Interés por el intercambio con países no-hispanohablantes

Entre los países no-hispanohablantes mencionados (en forma de respuesta abierta), se encuentra Finlandia en primer lugar con el $20.1 \%$ de las menciones; seguido por Francia (18\%), Estados Unidos (9.5\%), Alemania (8.5\%), Inglaterra $(8.5 \%$ ) y varios otros países con proporciones menores, como se puede ver en la Figura 8. 


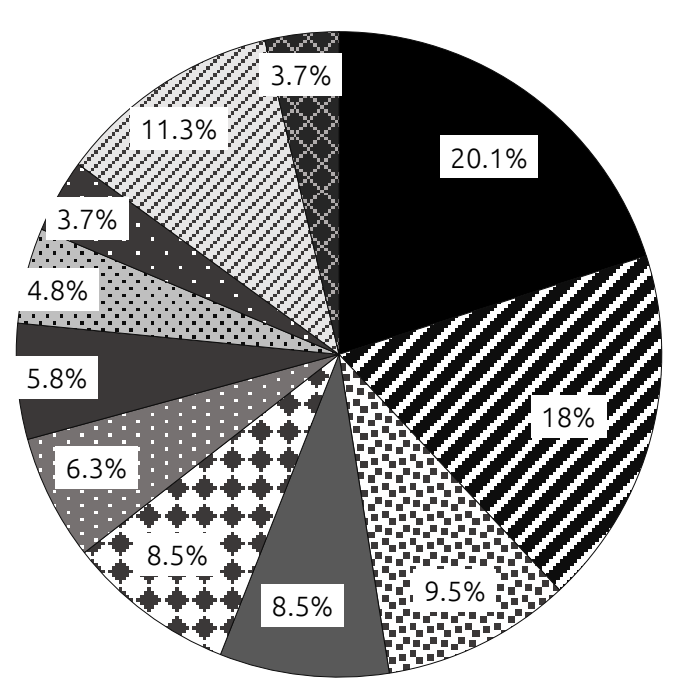

- Finlandia

- Francia

USA

$\square$ Alemania

$\checkmark$ Inglaterra

- Canadá

- Japón

Italia

- China

$\square$ Otros doce

países

No contestó

Figura 8. País no-hispanohablante preferido como primera opción

Cabe indicar que el alto interés por participar en algún programa de intercambio internacional refleja (de manera lógica) la importancia atribuida al contacto con "lo extranjero"; sin embargo, lo que se considera importante y deseable no necesariamente se puede conocer de primera mano, por lo que es importante tomar en cuenta las experiencias personales concretas que los normalistas hayan o no tenido con "lo extranjero".

\subsection{Experiencias concretas con "lo extranjero" en el contexto de la formación magisterial}

La figura 9 demuestra las proporciones porcentuales del nivel de contacto que los estudiantes normalistas dicen haber tenido con maestros visitantes del extranjero desde que ingresaron a la ENSM.

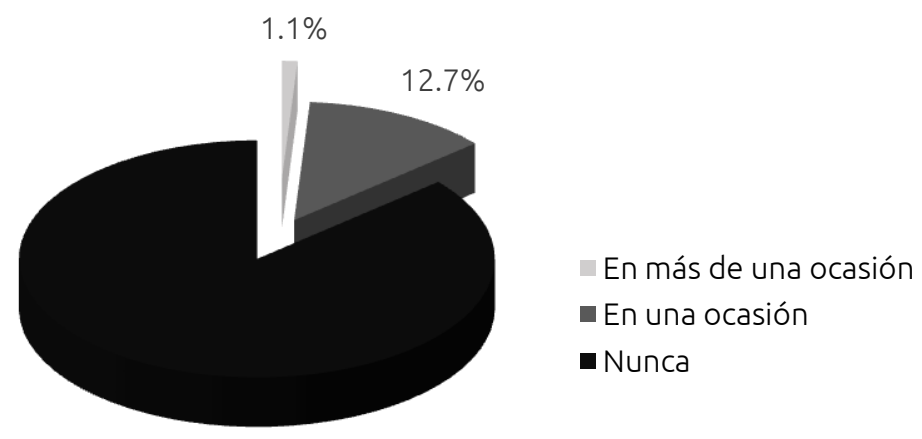

$86.2 \%$

Figura 9. Nivel de contacto con maestros visitantes del extranjero

Al revisar la figura de sectores correspondiente, se observa que la mayoría de los normalistas (86.2\%) nunca ha tenido contacto con maestros visitantes del extranjero. 
Como se puede ver en la figura 10, se obtuvieron resultados parecidos respecto al nivel de recepción de información sobre la existencia de programas de intercambio internacional de maestros - también desde que ingresaron a la ENSM.

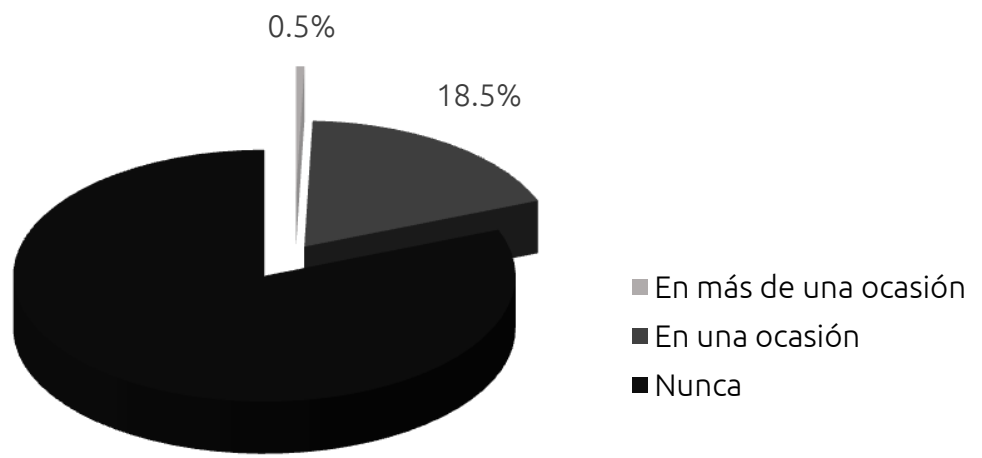

$81 \%$

Figura 10. Nivel de información recibida sobre programas de intercambio internacional de maestros

De hecho, el $81 \%$ de los estudiantes normalistas indicó nunca haber sido informado sobre programas de intercambio para (futuros) maestros. ${ }^{4}$

Por último, la proporción de encuestados que dice haber participado en algún programa de intercambio internacional (ver figura 11) corresponde al 1.1\%; el resto de los estudiantes normalistas, es decir, cerca de 10 de cada 10 encuestados, revelaron nunca haber participado en algún programa de intercambio internacional -en esta ocasión no sólo desde su ingreso a la ENSM, sino desde su educación básica.

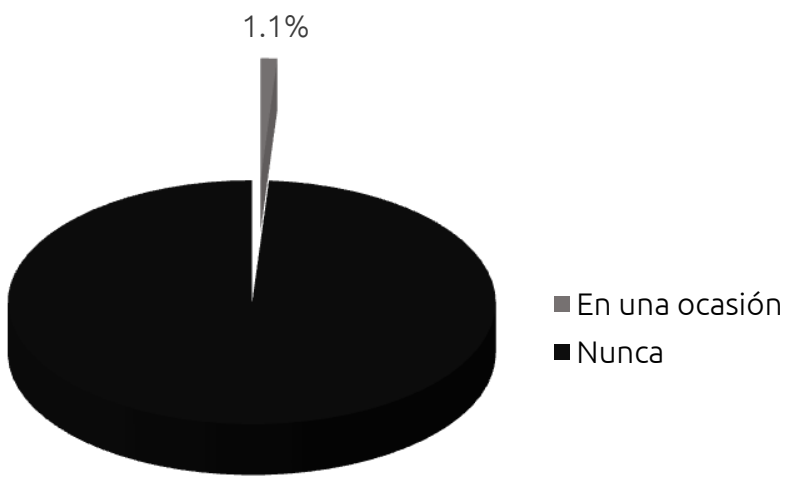

$98.9 \%$

Figura 11. Nivel de participación en programas de intercambio internacional

Con base en los resultados empíricos expuestos en la presente sección, y tomando en cuenta el amplio contexto nacional presentado en la introducción de este artículo, es oportuno finalizar con una conclusión sintetizada y prospectiva.

\footnotetext{
${ }^{4}$ Al preguntar específicamente si se había escuchado sobre el programa de intercambio "INTERJOM" entre México y España -es decir, con el país hispanohablante más frecuentemente mencionado como destino deseado (ver figura 6)- el 98.9\% de los encuestados respondió que no.
} 


\section{Discusión y conclusiones}

Las respuestas de los estudiantes de la ENSM ofrecen un panorama en el que se observan, por un lado, altos niveles de importancia atribuidos al contacto con "lo extranjero" como parte integral de la formación magisterial, confirmados por altos niveles de interés por participar en algún programa de intercambio internacional y -por otro lado- la falta de (información sobre) programas de intercambio y de experiencias con "lo extranjero" realmente vividas. Es así que, de manera notable, los resultados de la encuesta a futuros maestros mexicanos de secundaria reflejan frustraciones asociadas a un analfabetismo intercultural importante a nivel curricular y sociocultural.

Contrario a lo que los principales medios de comunicación han sugerido en los últimos años - en lugar de preguntarnos en dónde están los estudiantes normalistas motivados y hambrientos de conocimiento, deberíamos cuestionarnos: ¿En dónde está la voluntad política para proveer a las maestras y maestros del país oportunidades de contacto con "lo extranjero"?- y en consecuencia: ¿En dónde están los planes curriculares, las provisiones institucionales, los programas de gobierno, la infraestructura y los recursos humanos orientados a la internacionalización, la interculturalidad y el desarrollo multiperspectivo de la formación magisterial?

Como hemos señalado en la introducción de este artículo, los maestros son pieza clave para la creación de entornos escolares de diálogo y aprendizaje interculturales socioculturalmente justos e incluyentes. Por lo que es de fundamental importancia que se conozcan y tomen en cuenta sus propias perspectivas perceptuales. Aunque nuestro análisis se basó en una muestra de sólo 189 personas, se espera que las percepciones, los intereses y las experiencias de este conjunto de futuros maestros puedan servir como punto de referencia para el desarrollo de (futuras investigaciones sobre) la formación magisterial intercultural inicial y continua -dentro y más allá del contexto específico de la ENSM.

\section{Referencias}

Balda, N. (2015). La formación de maestros en Bolivia: dos visiones y nuevas perspectivas. Praxis Educativa, 19(2), 27-33.

Constitución Política de los Estados Unidos Mexicanos (2016). Artículo 2, Párrafo 2 [consultada el 14 de junio de 2016].

Ducoing, P. (Coord.) (2013). La Escuela Normal. Una mirada desde el otro. Ciudad de México: IISUE-UNAM.

Ducoing, P. (2007). La educación secundaria. Un nivel demandante de especificidad y un objeto de estudio emergente. Revista Mexicana de Investigación Educativa, 12(32), 7-36.

Fernández, M. T., Torres, E. y García, C. (2016). Creencias sobre la educación intercultural y prácticas de enseñanza de profesores de secundaria de la población indígena yaqui. Perfiles Educativos, 38(152), 109127.

Hecht, A. C. (2015). Educación intercultural bilingüe en Argentina. Un panorama actual. Ciencia e Interculturalidad, 16(1), 20-30.

Leiva, J. (2006). La formación intercultural del docente: reflexiones en el ámbito de la formación inicial y permanente. Revista Comunicación, 15(1), 57-62.

Paladino, M. (2015). Las complejas tramas de la educación escolar indígena en Brasil: discursos de interculturalidad y apropiaciones indígenas de la escuela. Relaciones. Estudios de Historia y Sociedad, 36(141), 47-73. 
Pérez-Silva, S. y Luis, J. (2015). Creciente importancia de la formación del docente de la educación superior en un mundo de cambio. Reflexiones del padre Félix Varela. Revista Universidad y Sociedad, 7(1), 97-101.

Pöllmann, A. (2016). Habitus, reflexividad y la realización de capital intercultural: El potencial (no aprovechado) de la educación intercultural. Cultura y Representaciones Sociales, 11(21), 55-78.

Pöllmann, A. (2015). Cultura, lectura y deporte: Percepciones, prácticas, aprendizaje y capital intercultural. Encuesta Nacional de Cultura, Lectura y Deporte. En J. Flores y A. Morales (Coords.), Los mexicanos vistos por sí mismos. Los grandes temas nacionales. Inventario de México en 2015 (pp. 153-163). México: IIJ-UNAM.

Pöllmann, A. (2014). Capital intercultural: Hacia la conceptualización, operacionalización e investigación empírica de un marcador de distinción sociocultural en ascenso. Cultura y Representaciones Sociales, 9(17), 54-73.

Pöllmann, A. (2009). Formal education and intercultural capital. Towards attachment beyond narrow ethno-national boundaries? Educational Studies, 35(5), 537-545.

Pöllmann, A. y Sánchez-Graillet, O. (2015). Cultura, lectura y deporte. Percepciones, prácticas, aprendizaje y capital intercultural. Encuesta Nacional de Cultura, Lectura y Deporte. Ciudad de México: IIJ-UNAM.

Santoro, N. (2014). 'If I'm going to teach about the world, I need to know the world': developing Australian pre-service teachers' intercultural competence through international trips. Race Ethnicity and Education, 17(3), 429-444.

Secretaría de Educación Pública. (2000). Licenciatura en educación secundaria. Documentos básicos. Programa para la transformación y el fortalecimiento académicos de las Escuelas Normales. Ciudad de México: SEP.

Turra, O. y Ferrada, D. (2016). Formación del profesorado en la lengua y cultura indígena: una histórica demanda educativa en contexto Mapuche. Educação e Pesquisa, 42(1), 229-244.

Villalta, M. A. (2016). Educación intercultural en Latinoamérica: Análisis de las investigaciones de campo en la región. Psicoperspectivas, 15(1), 118-131. 